\title{
The Silicon-Tungsten Tracker of the DAMPE Mission
}

\author{
X. Wu ${ }^{1 *}$, G. Ambrosi $i^{2}$, R. Asfandiyarov ${ }^{1}$, P. Azzarello ${ }^{1}$, P. Bernardini ${ }^{3,4}$, B. Bertucci $i^{2,5}$, \\ A. Bolognini ${ }^{2,5}$, F. Cadoux ${ }^{1}$, M. Caprai ${ }^{2}$, I. De Mitri ${ }^{3,4}$, Y. Dong ${ }^{6}$, M. Duranti ${ }^{2,5}$, R. Fan $^{6}$, \\ P. Fusco ${ }^{7,8}$, V. Gallo', F. Gargano ${ }^{7}$, D. Guo ${ }^{6}$, C. Husi ${ }^{1}$, M. Ionica ${ }^{2,5}$, G. Ke ${ }^{6}$, D. La

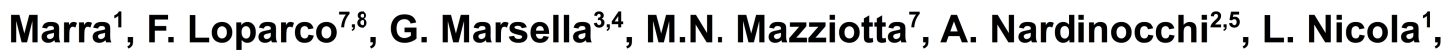 \\ G. Pelleriti ${ }^{1}$, W. Peng ${ }^{6}$, V. Postolache ${ }^{2}$, M. Pohl ${ }^{1}$, R. Qiao ${ }^{6}$, A. Surdo ${ }^{4}$, A. Tykhonov ${ }^{1}$, \\ S. Vitillo', H. Wang ${ }^{6}$, M. Weber ${ }^{1}$, D. Wu ${ }^{6}$, F. Zhang ${ }^{6}$ \\ 'Département de Physique Nucléaire et Corpulsculaire, University of Geneva, Geneva, Switzerland \\ ${ }^{2}$ Istituto Nazionale di Fisica Nucleare Sezione di Perugia, Perugia, Italy \\ ${ }^{3}$ Dipartimento di Matematica e Fisica "E. De Giorgi", Universita' del Salento, Lecce, Italy \\ ${ }^{4}$ Istituto Nazionale di Fisica Nucleare Sezione di Lecce, Lecce, Italy \\ ${ }^{5}$ Dipartimento di Fisica e Geologia, Università di Perugia, Perugia, Italy
}

${ }^{6}$ Istitute of High Energy Physics, Chinese Academy of Aciences, Beijing, China

'Istituto Nazionale di Fisica Nucleare, Sezione di Bari, Bari, Italy

${ }^{8}$ Dipartimento di Fisica “M. Merlin” dell'Università e del Politecnico di Bari, Bari, Italy

\begin{abstract}
The DArk Matter Particle Explorer (DAMPE) is a high energy astroparticle satellite mission designed to detect electron, photon and cosmic rays with high precision for Dark Matter search, cosmic ray flux and composition measurement and gamma-ray astronomy. One of the key components of the DAMPE payload is the Silicon-Tungsten Tracker (STK), consisting of 6 tracking planes, each plane is made of 2 orthogonal layers of single-sided silicon micro-strip detectors. Three layers of $1 \mathrm{~mm}$ thick tungsten plates are interleaved with the tracking planes to serve as photon converter. Besides precise track reconstruction for charge particles and converted photons, the STK will also measure the charge of the incoming cosmic ray, and provide pre-shower information to improve particle identification.
\end{abstract}

After intensive design, prototyping, test and production efforts by the STK collaboration, the construction of the STK has been completed. An Engineering and Qualification Model (EQM) has been produced in April 2014 and passed space qualification tests, as well as extensively tested at with particle beams at CERN. The Flight Model (FM) has been produced in April 2015 and successfully integrated into the DAMPE payload in June 2015, after passing the environmental acceptance test. The DAMPE satellite is scheduled to be launched at the end of 2015.

The 34th International Cosmic Ray Conference

30 July- 6 August, 2015

The Hague, The Netherlands

*Speaker 


\section{Introduction}

The DArk Matter Particle Explorer (DAMPE)[1] is a high energy astroparticle satellite mission designed to detect high energy gamma rays and cosmic rays with high precision. The main science objectives are 1) the search for Dark Matter decay or annihilation signatures, 2) the measurement of cosmic ray flux and composition, and 3) gamma-ray astronomy. DAMPE is of the five scientific space science missions planned within the framework of the Strategic Pioneer Program on Space Science[2] of the Chinese Academy of Sciences (CAS). The design, prototyping, production, test and qualification of the DAMPE payload has been carried out by collaborating institutes ${ }^{1}$ from China, Switzerland and Italy.

The performance goal of the DAMPE detector is to measure precisely the electron and photon spectrum between $5 \mathrm{GeV}$ to $10 \mathrm{TeV}$, and cosmic ray flux and chemical composition between $10 \mathrm{GeV}$ to above $100 \mathrm{TeV}$, with reasonably large geometrical acceptances, $\sim 0.3 \mathrm{~m}^{2} \mathrm{sr}$ for electrons/photons, and $\sim 0.2 \mathrm{~m}^{2} \mathrm{sr}$ for cosmic rays, using the technologies of a thick total absorption calorimeter, and a precise tracker with integrated photon converters. The DAMPE detector, as shown in Figure 1, consists of 4 subsystems: the plastic scintillator strips detector (PSD), the silicon-tungsten tracker-converter (STK), the BGO imaging calorimeter (BGO), and the neutron detector (NUD).

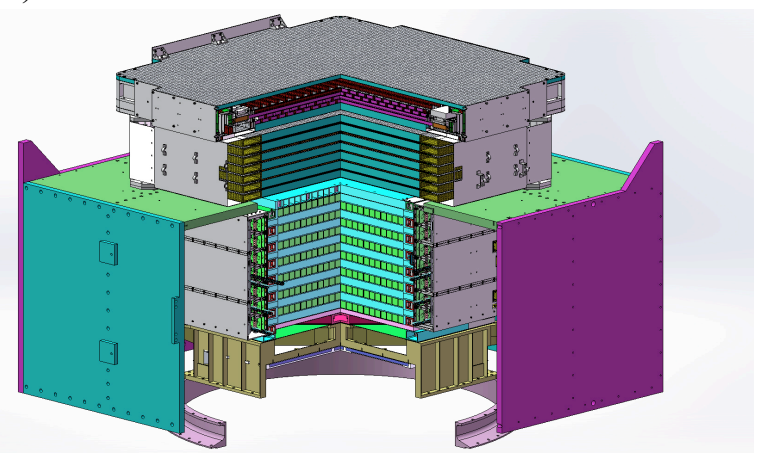

Figure 1: Sketch of the DAMPE detector

The PSD consists of one double layer (one $\mathrm{x}$ and one $\mathrm{y}$ ) of scintillating strips detector made of scintillating strips of $1 \mathrm{~cm}$ thick, $2.8 \mathrm{~cm}$ wide and $82 \mathrm{~cm}$ long. The strips are staggered by $0.8 \mathrm{~cm}$ in a layer, thus fully covers an area of $82 \mathrm{~cm}$ by $82 \mathrm{~cm}$. The PSD serves as anticoincidence detector for photon identification, as well as charge detector for cosmic rays. The design specification is a position resolution of $6 \mathrm{~mm}$, and a charge resolution of 0.25 for $Z=1$ to 20. The STK is the main subject of this report and details will be given in following sections. The calorimeter is made up of 14 layers of BGO bars in a hodoscopic arrangement. Each BGO bar is $2.5 \mathrm{~cm}$ by $2.5 \mathrm{~cm}$ in cross section and $60 \mathrm{~cm}$ in length, making it the longest BGO crystals ever produced. The bars are readout at both ends with PMTs, each PMT is readout from 3 dynodes $(2,5,8)$ to extend the dynamic range. The total thickness of the calorimeter is equivalent to 31 radiation lengths and 1.6 interaction lengths. An excellent electromagnetic energy resolution of $1.5 \%$ above $100 \mathrm{GeV}$, and a very good hadronic energy resolution of better than $40 \%$ above $800 \mathrm{GeV}$ can be expected. The neutron detector (NUD) consists of $161 \mathrm{~cm}-$ thick boron-doped plastic scintillator plates of $19.5 \mathrm{~cm}$ by $19.5 \mathrm{~cm}$ large each read out by a

${ }^{1}$ PMO-USTC-IMP-IHEP-NSSC-UNIGE-PERUGIA-BARI-LECCE 
PMT. The purpose of the NUD is to detect delayed neutron resulting from a hadron shower in order to improve the electron/proton separation power, which should be $10^{5}$ overall.

\section{The Silicon-Tungsten Tracker}

Silicon micro-strip tracker is a proven space technology that is used successfully in space experiments, eg. PAMELA, AGILE, Fermi and AMS. The tracker-converter concept, in which tungsten foils are inserted between silicon tacking layers, has been employed in AGILE and Fermi and proved to be an excellent technique to detect high-energy photons with high efficiency and precision. An incoming photon converts to an electron-positron pair in the tungsten converter, which can then be detected by subsequent silicon layers. Multiple scattering of charged particles can be mitigated by spreading the tungsten to several foils, and for highenergy particles $(>5 \mathrm{GeV})$, the effect is negligible for tungsten foils of a few millimeters thickness. The STK combines the features of the AMS tracker and the Fermi/AGILE trackerconverters to perform three important tasks: charge track reconstruction, photon detection, and charge measurement of cosmic rays. The STK is made of 6 tracking planes each consists of two layers of single-sided silicon strip detectors measuring the two orthogonal views perpendicular to the pointing direction of the apparatus. Three layers of tungsten plates of $1 \mathrm{~mm}$ thick are inserted in front of tracking layer 2, 3 and 4 for photon conversion. Figure 2 shows an exploded view of the STK internal structure (left panel) and a picture of the Engineering and Qualification Model of the STK (right panel).
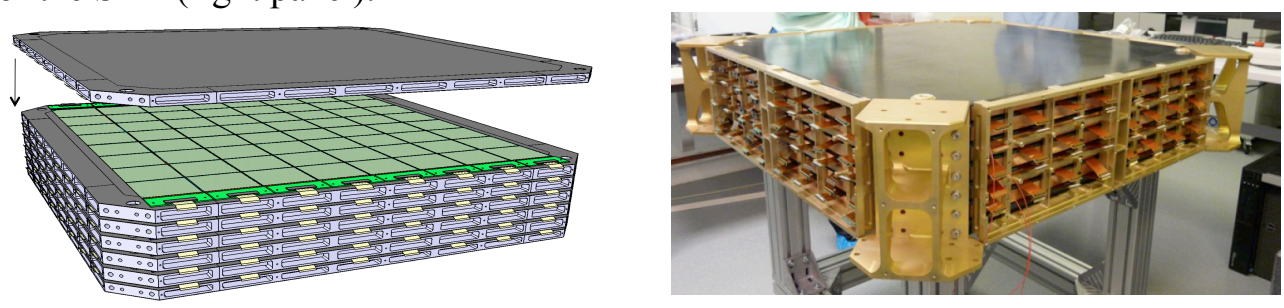

Figure 2: Exploded view of the STK internal structure (left). A picture of the STK EQM (right).

The STK uses single-sided AC-coupled silicon micro-strip detectors. The sensor is $9.5 \mathrm{~cm}$ by $9.5 \mathrm{~cm}$ in size, $320 \mu \mathrm{m}$ thick, and segmented into 768 strips with a $121 \mu \mathrm{m}$ pitch. Only every other strip is readout but since analogue readout is used the position resolution is better than 80 $\mu \mathrm{m}$ for most incident angles, thanks to the charge division of floating strips. The photon angular resolution is expected to be around $0.2^{\circ}$ at $10 \mathrm{GeV}$. The high dynamic range of the analog readout electronics of the STK allows to measure the charge of the incident cosmic rays with high precision. The full tracker uses 768 sensors, equivalent to a total silicon area of $\sim 7 \mathrm{~m}^{2}$.

\subsection{The Silicon Sensor and the Silicon Ladder}

The single-sided AC-coupled silicon strip detectors (SSSD) were manufactured by Hamamatsu Photonics, using the same sensor geometry used by the AGILE mission, but with some noticeable differences in thickness, bulk resistivity and backplane metallisation. The backplane metallisation is gold instead of aluminium which improves the backplane contact. The bulk resistivity is $5-8 \mathrm{k} \Omega \mathrm{cm}$, which allows to operate the $\mathrm{STK}$ at $80 \mathrm{~V}$. The body capacitance is $0.4 \mathrm{pF} / \mathrm{cm}$, the inter-strip capacitance is $0.85 \mathrm{pF} / \mathrm{cm}$, and the total strip capacitance is 2.1 $\mathrm{pF} / \mathrm{cm}$. Leakage currents at $150 \mathrm{~V}$ of the 865 sensors ( 768 needed for the STK) delivered have 
been measured at the manufacturer, as shown in the left panel of Figure 3. The average value is $116 \mathrm{nA}$, well below the specification of $900 \mathrm{nA}$.
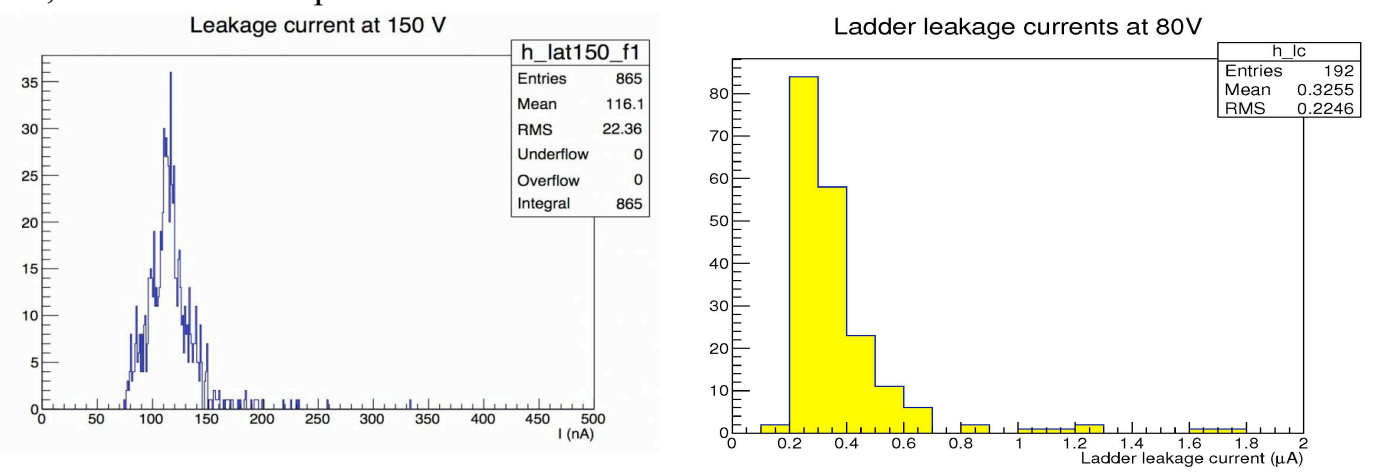

Figure 3: Leakage currents of 865 silicon strip sensors measured at $150 \mathrm{~V}$ at the manufacturer (left) and those of 192 ladders installed in the final Flight Model of the STK (right).

The basic detection unit of the STK is the "Silicon Ladder", formed by four silicon sensors glued to a bias circuit (flexible PCB) then the strips wire-bonded in series to form a ladder of about $40 \mathrm{~cm}$ long and $9.5 \mathrm{~cm}$ wide, shown in the left bottom panel of Figure 4 . The sensor bias circuit is integrated with the frontend electronics board, as well as the readout flexible cable (the "pigtail"), as shown in the left top panel of Figure 4. The integrated circuit, called the Tracker Frontend Hybrid (TFH) uses a novel approach combining flexible and rigid PCB technology, thus minimizing the assembly procedure and the usage of connectors.
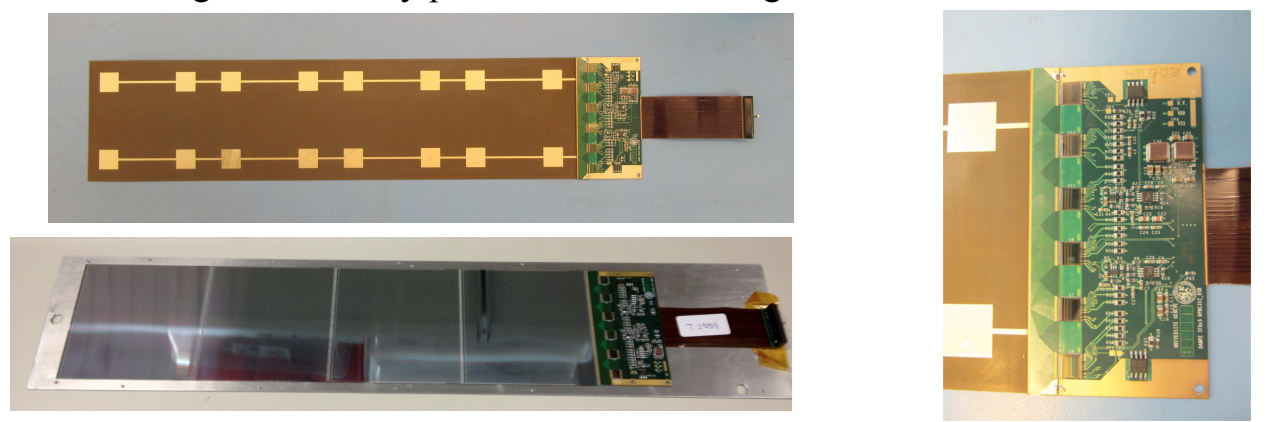

Figure 4: A STK Tracker Frontend Hybrid (TFH) (left top), a STK Silicon Ladder (left bottom), and the details of the TFH (right).

The silicon strips are read out by the 64-channel analogue low power high dynamic range VA140 ASIC manufactured by Gamma Medica-Ideas AS, based in the readout chip developed for the AMS silicon tracker. Six VA140 chips are housed per TFH to read out the 384 readout channels of a silicon ladder. In addition to the signal readout, the TFH contains also a circuit to distribute bias voltage to the silicon sensors, as well as circuits for temperature sensors. The right panel of Figure 4 shows the circuit details of the TFH.

The 6 VA140 chips on a TFH are read out in 2 groups of 3 in parallel. The charges of the 192 strips connected to the 3 chips in a group are read out serially through the "pig tail" of the TFH into an Analogue-to-Digital-Converter (ADC) on the Tracker Readout Board (TRB), where they are digitized and zero suppressed. FPGAs on the TRB then perform the cluster finding and send the clusters to the DAMPE DAQ system. The TRB system was designed and produced in IHEP, Beijing. Each TRB consists a set of 3-board (ADC Board, FPGA Board and Power Board) assembly. 
The assembled TFH were thermal screened according to space environmental requirements in a setup specially designed for this purpose using a thermal chamber. The screening included 2 power on-off cycles and 17 power-on cycles between $-30^{\circ} \mathrm{C}$ and $60{ }^{\circ} \mathrm{C}$; the full process took 7 days. TFH passed the screening test were used for ladder production at University of Geneva and INFN Perugia. Produced ladders were measured for the important 4sensor alignment with metrology machine. Thanks to the precise custom made ladder production jigs, the alignment precision of produced ladders was about $4 \mu \mathrm{m}$ on average, much better than the required $40 \mu \mathrm{m}$, as shown in Figure 5, for a sample of 192 ladders.
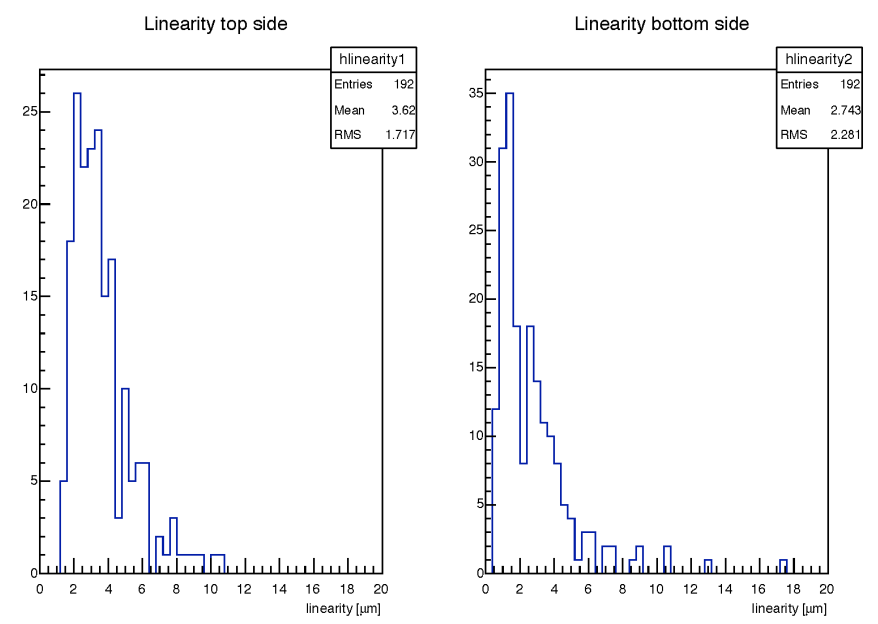

Figure 5: Alignment error of the top (left) and bottom (right) side of 192 ladders

The last step of single ladder quality assurance was a 12-hour cosmic ray test and the signal to noise ratio of cluster charges from minimum ionizing particles (MIPs) are checked. On average the $\mathrm{S} / \mathrm{N}$ is about 15 , excellent for the application of the STK. All 192 ladders needed for the STK Flight Model have been produced by the end of March 2015.

\subsection{The STK Tracker Plane and the Tracker Assembly}

Sixteen silicon ladders, arranged in two rows of eight, are glued to the surface of a support tray to cover an area of about $80 \mathrm{~cm}$ by $80 \mathrm{~cm}$, forming a silicon layer (right panel of Figure 8). A support tray is made of an aluminium honeycomb structure sandwiched between two Carbon Fibre Reinforced Polymer (CFRP) face sheets of $0.6 \mathrm{~mm}$ thick each, forming a light but rigid structure that can sustain the vibration and the acceleration of a rocket launch. Tungsten plates of $1 \mathrm{~mm}$ thick are integrated into the support trays of the second, third and forth tracker planes in such a way that the converter is located immediately above a tracking layer in order to ensure good conversion detection efficiency. Figure 6 shows the production of a support tray with $\mathrm{W}$ plates at Composite Design Sàrl. After fabrication each tray with W was scaned by X ray at CERN to check the alignment of the $\mathrm{W}$ plates with respect to the 4 corners.
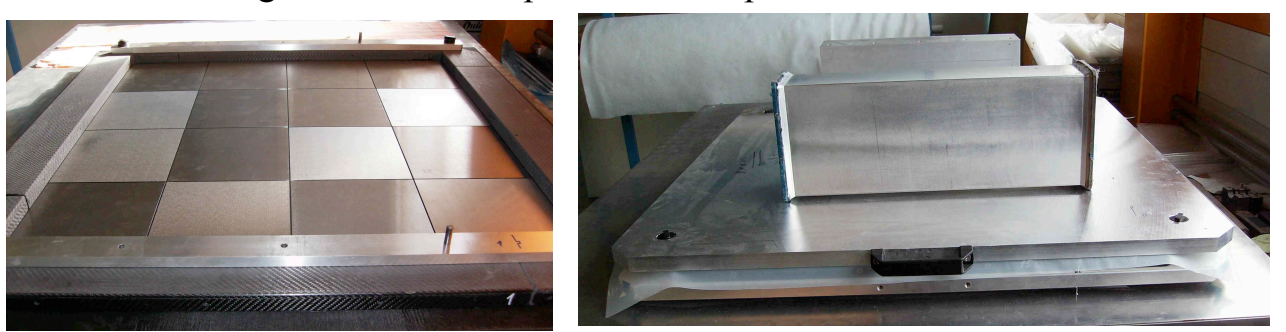

Figure 6: Tungsten plates being glued in a support tray (left); Final gluing of a support tray (right). 
A support tray is covered in both surfaces with silicon ladders to form a tracker plane, except for the top and the bottom trays, which have only one surface covered. The gluing of ladders on the tracker plane was carried out at the University of Geneva, with custom made jigs on a rotating stand, two ladders at a time. Figure 7 shows the gluing of ladders to a support tray and the metrology measurement of a fully loaded plane.
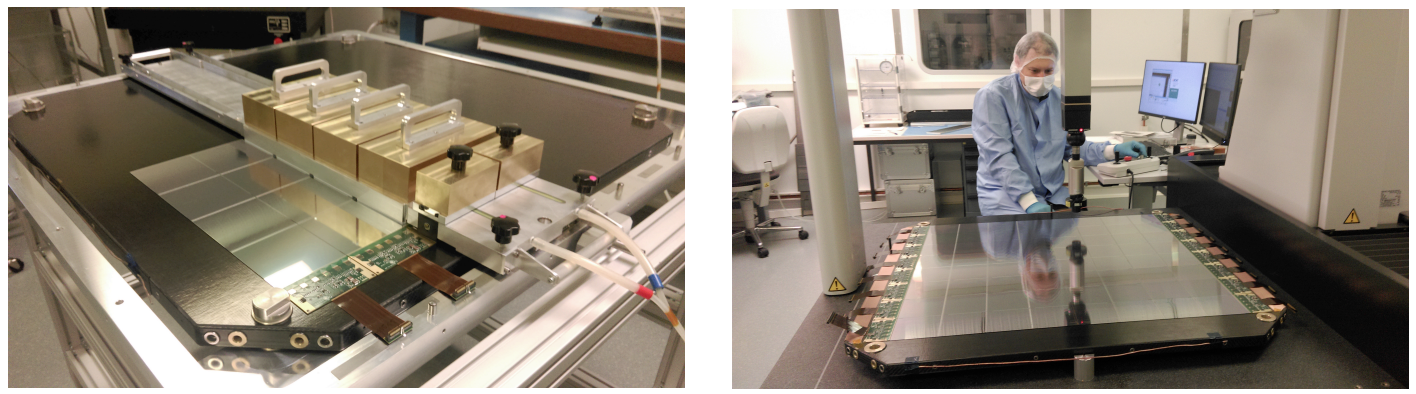

Figure 7: Gluing ladders to a support tray (Left); Metrology measurement of a completed tracker plane (right).

Once a plane is assembled, all ladders on the plane are measured again for noise and leakage current. The right panel of Figure 3 shows the measured leakage currents at $80 \mathrm{~V}$ of 192 ladders glued to the 7 planes. The excellent quality of the silicon sensors has been maintained through the ladder production and plane assembly procedures.

Tracker planes are stacked on top of each other to form the full tracker. The silicon ladders on the bottom surface of a tracker plane and those on the top surface of the plane below form an $\mathrm{x}-\mathrm{y}$ tracking plane, the distance between the $\mathrm{x}$ and the $\mathrm{y}$ layer of a tracking plane is about $3 \mathrm{~mm}$. The stack of the 7 tracker planes forms 6 tracking planes that provide 12 coordinate measurements $(6 \mathrm{x}$ and $6 \mathrm{y})$ when a charged track traverses the STK. Figure 8 shows the schematic of the STK mechanical structure (left panel) and the assembly of the STK Flight Model in progress, with all but the top tracker plane stacked (right panel).
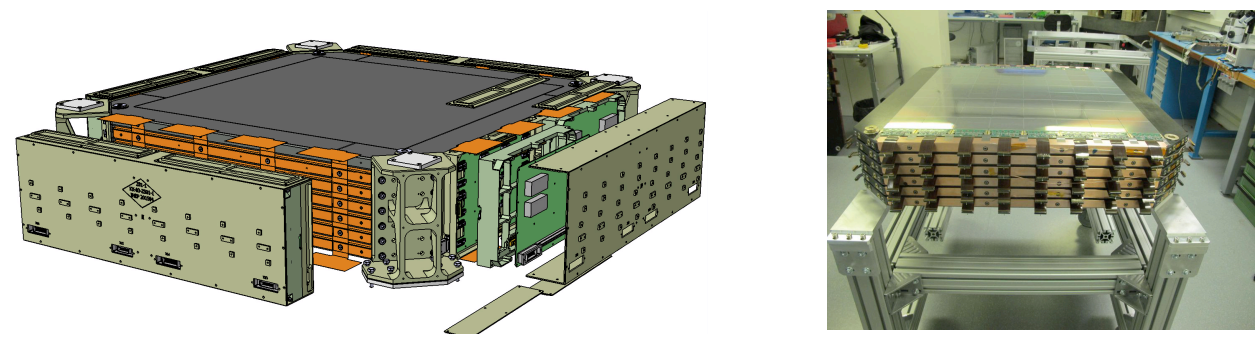

Figure 8: Schematic of the STK mechanical structure (left) and the STK FM being assembled (right).

To minimize power consumption, low power components are used as much as possible. The power consumption of each ladder is about $116 \mathrm{~mW}$ and the total for the STK is about $90 \mathrm{~W}$. The STK uses a passive cooling systems of heat conductors: copper vias and bands on the TFH, pyrolytic graphite sheets (PGS) that connects from TFH to copper straps, which in turn transfers heat to the aluminium radiators on the outside of the STK. The thermal management system of the satellite uses heat pipes to keep the radiator at a constant temperature at $10^{\circ} \mathrm{C}$.

\section{The Construction and Test of the Engineering and Qualification Model (EQM)}

The main design concepts of the mechanical support structure and thermal management have been qualified with the EQM in a series of space environmental qualification tests, including vibration, acceleration, shock, thermal cycling and thermal vacuum tests. Figure 9 
shows the EQM being prepared for the vibration tests (left panel) and for the thermal vacuum tests (right panel) at SERMS srl in Italy. The EQM is a full size tracker model that has the same thermal, mechanical and data interface to the satellite as the final Flight Model (FM). However only 26 functioning silicon ladders have been assembled on the EQM, the remaining 166 being mechanical ladders with the same mechanical and thermal properties as the real silicon ladders.
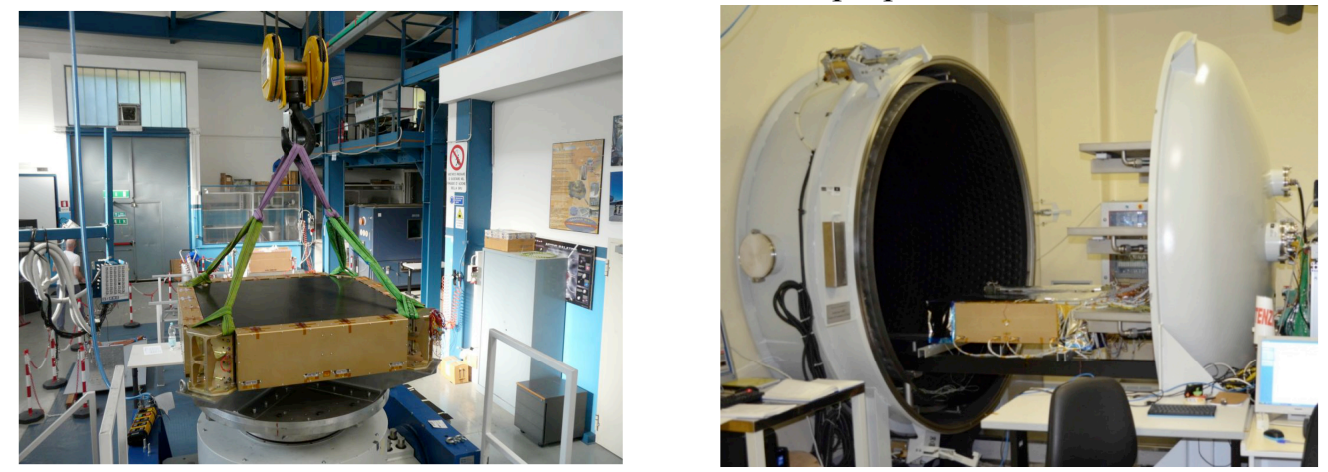

Figure 9: The STK EQM being installed for vibration (left) and thermal vacuum tests in SERMS, Terni, Italy.

The EQM has also been integrated into the DAMPE prototype full detector and tested extensively with particle beams at CERN PS and SPS during 2014 and 2015. Data with protons, electrons, muons, photons and nuclear fragments have been taken and are being analysed to assess the detector performance. Figure 10 shows the DAMPE prototype detector in the CERN PS T9 beam line during the 2014 test period (left panel), and an event display of a photon converted in the STK.
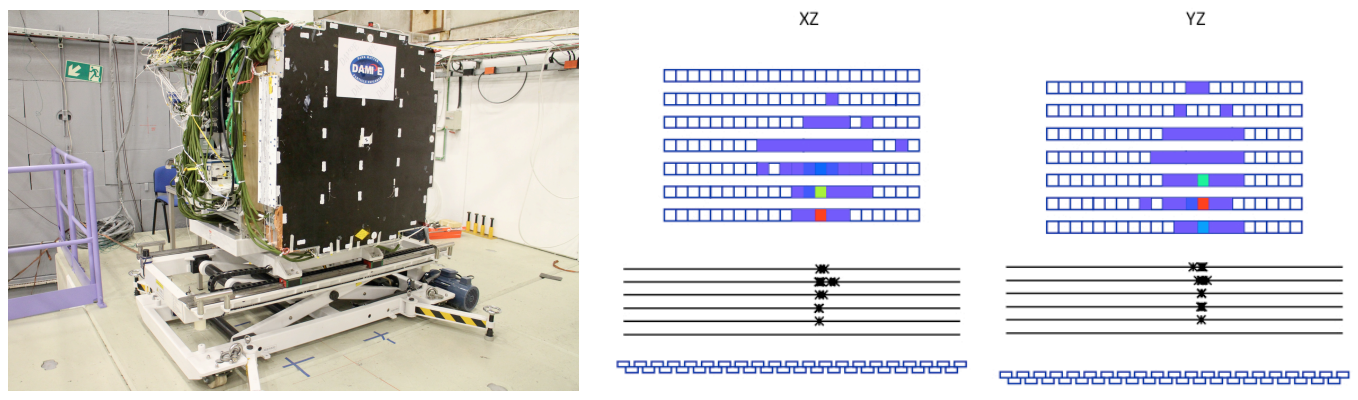

Figure 10: The DAMPE prototype detector in the PS T9 beam line(left); An event display of a photon converted in the STK (right).

\section{The Status of the STK Flight Model (FM)}

The STK Flight Model, to be used in the DAMPE payload, has been constructed in April 2015 in Geneva and tested with cosmic rays for several days, before being delivered to IHEP in Beijing. Acceptance level of vibration and thermal vacuum tests were conducted in Beijing in May 2015. In June 2015 the STK FM has successfully passed the DAMPE payload integration test and then the DAMPE satellite integration test. After 2 full months of intensive manipulations and tests the STK remains in excellent quality. At the end of the satellite integration test, the percentage of noisy channels (noise $>5$ ADC counts) remains below $0.3 \%$. Only $18(<0.03 \%)$ channels out of the 74382 need to be masked (see Figure 11). Currently integration and tests at the satellite level is progressing to meet the scheduled launch at the end of 2015 . 

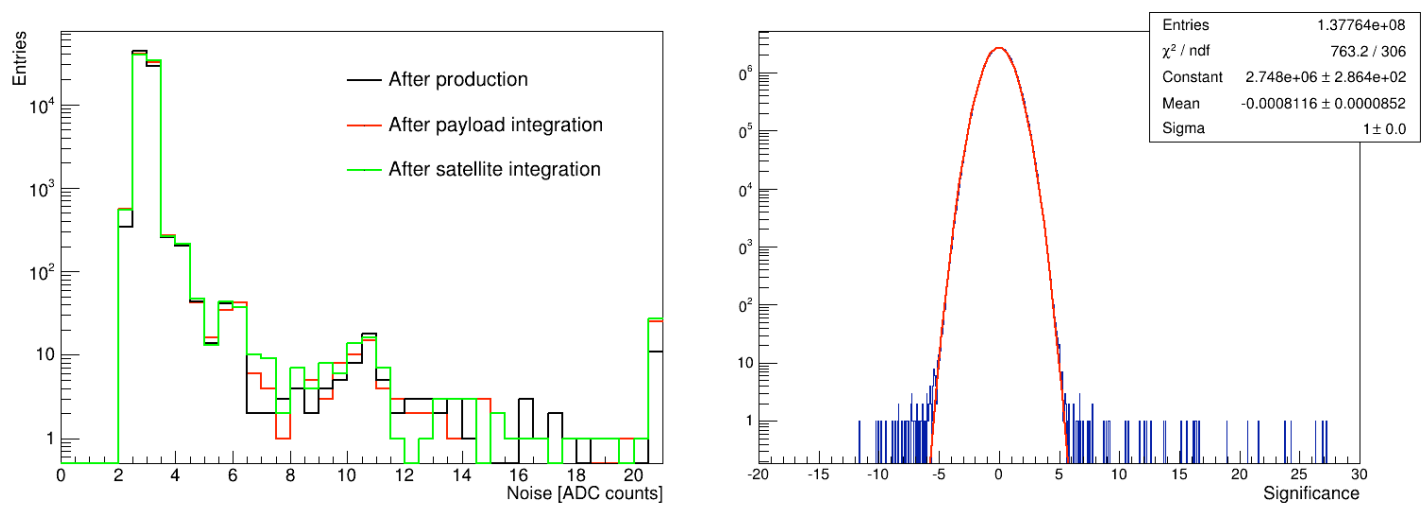

Figure 11: Measured noise of 73728 channels of the STK FM (left); Signal to noise ratio for a noise run after satellite integration of all channels. Some cosmic ray contamination in the noise run is visible (right).

\section{Conclusions}

The Silicon-Tungsten Tracker (STK) of the DAMPE mission is based on robust technology of single-sided silicon strip detectors with analog readout. It will play crucial roles in charge track reconstruction, gamma-ray detection, cosmic ray charge measurement, and overall particle identification. After 2 years of intensive design, prototyping, testing and production efforts, an Engineering and Qualification Model has been completed and qualified and a Flight Model (FM) has been produced and passed acceptance test. The quality of the FM is excellent and meets the design specification.

\section{Acknowledgement}

The authors wish to express their gratitude to M. Prest and E. Vallazza of the AGILE Silicon Tracker collaboration for fruitful discussions on tracker design and for kindly allowing us to use the AGILE silicon sensor geometry. The generosity of CERN for providing beam time allocation and technical assistance at the PS and SPS beam lines, as well as general logistical and technical support, including the bonding of several ladders, is acknowledged. This work is supported by the Chinese Academy of Sciences, the Swiss National Science Foundation and INFN, Italy.

\section{References}

[1] J. Chang, in The 7th international workshop "Dark Side of the Universe (DSU 2011)" (2011). (http://kitpc.itp.ac.cn/dsu2011/slides/DSU2011-J.Chang.ppt)

[2] Science Magazine 332 (6032): 904, 20 May 2011. 